\title{
Modelos de Previsão do Tom Emocional de Usuários em Comunidades de Saúde Mental no Reddit
}

\author{
Bárbara Silveira, Ana Paula Couto da Silva, Fabricio Murai \\ ${ }^{1}$ Departamento de Ciência da Computação \\ Universidade Federal de Minas Gerais (UFMG) - Belo Horizonte, MG - Brazil \\ \{barbarasilveira, ana.coutosilva, murai\}@dcc.ufmg.br
}

\begin{abstract}
The rise in the number of people afflicted by mental health problems has placed these disorders among the main public health problems worldwide. As a result, user activity in communities related to mental health in online social networks has spiked. Here we analyze how interactions through posts and comments influence the emotional state of users of mental health communities on Reddit, investigating whether seeking help on these networks results in changes in the feelings expressed by users over time. Our results show that the state of users changes over time, and that in $68 \%$ of cases the change is positive. We also propose predictive models to capture the variation of the emotional tone.
\end{abstract}

Resumo. O crescimento do número de pessoas atingidas por problema de saúde mental colocou tais distúrbios entre os principais problemas de saúde pública em todo o mundo. Como resultado, aumentou-se a procura por comunidades sobre saúde mental em redes sociais online. Neste artigo, analisamos como as interações através de posts e comentários influenciam no estado emocional de usuários de comunidades de saúde mental no Reddit, verificando se a busca por auxílio nestas redes resulta em mudanças nos sentimentos expressos pelos usuários ao longo do tempo. Nossos resultados mostram que o estado dos usuários muda ao longo do tempo, e que em $68 \%$ dos casos a mudança é positiva. Também propomos modelos preditivos para capturar a variação do tom emocional.

\section{Introdução}

Dados da Organização Mundial de Saúde (OMS) mostram que a cada 40 segundos uma pessoa morre devido ao suicídio, sendo esta a segunda causa de morte entre jovens entre 15 e 29 anos $^{1}$. Diversos fatores podem resultar nesta situação extrema, dentre eles, transtornos mentais, como a ansiedade, a depressão e a bipolaridade. Para citar algumas estatísticas, a ansiedade atinge 264 milhões de indivíduos no mundo. $\mathrm{O}$ transtorno bipolar ${ }^{2}$ afeta cerca de 60 milhões de pessoas e, muitas vezes, este problema pode ser confundido com um caso de depressão ou de ansiedade, levando a um tratamento inadequado. Apesar desses números preocupantes, muitas das pessoas que sofrem de distúrbios mentais não recebem tratamento. Segundo a OMS, a cada 4 pessoas, 3 não recebem qualquer tipo de

\footnotetext{
${ }^{1}$ https://www.who.int/docs/default-source/mental-health/suicide/live-lifebrochure.pdf?sfvrsn=6ea28a12 2 download $=$ true

${ }^{2}$ https: //www. who.int/en/news-room/fact-sheets/detail/ mental-disorders
} 
tratamento e $45 \%$ da população mundial vive em um país com menos de um psiquiatra para cada 100 mil habitantes ${ }^{3}$.

Muitas pessoas que precisam de apoio acabam não sendo tratadas devido a ausência de recursos para apoiá-la ou pelo estigma social associado aos transtornos mentais [Barney et al. 2006], o que pode gerar consequências irrevisíveis. Assim, novas ferramentas e tratamentos para auxiliar pessoas que passam por problemas relacionados à saúde mental estão sendo propostos nos últimos anos. Em especial, podemos citar as redes sociais, as quais são facilmente acessadas a baixo custo pela maioria das pessoas. O trabalho realizado por [De Choudhury 2013] mostra que o compartilhamento online de pensamentos, sentimentos e experiências com outras pessoas contribui com a melhoria do bem-estar dos indivíduos.

Neste contexto, o presente artigo analisa como as interações através de posts e comentários influenciam o estado emocional dos usuários de comunidades de saúde mental no Reddit ${ }^{4}$, representado pelo tom emocional de suas mensagens. O Reddit é um site de fóruns com características de redes sociais: é composto por comunidades (subreddits), onde os usuários compartilham suas experiências e dúvidas sobre os mais diversos assuntos. Nesta rede, um usuário inicia uma thread ao publicar um post. Este post pode ser respondido por comentários de outros usuários ou mesmo daquele que escreveu o post. Comentários, por sua vez, podem ser respondidos por outros comentários. Dados de 2019 mostram que o Reddit é constituído por 430 milhões de usuários ativos por mês. Ao todo são 199 milhões de posts, 1,7 bilhões de comentários ${ }^{5}$.

Nosso trabalho tem dois objetivos principais: (1) compreender e medir como redes sociais online são utilizadas para apoiar na melhoria do tom emocional de usuários com problemas de saúde mental e (2) definir modelos que permitam acompanhar a evolução do tom emocional do usuário. A partir dos objetivos listados, nossas contribuições são:

1. Análise do Tom Emocional dos Usuários. Tom emocional (TE) é o sentimento (combinação do positivo e negativo) extraído através de um texto. Sabendo que existem usuários que procuram estas comunidades em busca de ajuda e outros que estão dispostos a ajudar, analisamos a variação do estado emocional de um usuário dentro de uma thread, verificando se os outros usuários influenciam no TE do usuário que iniciou a discussão. Como principais resultados, observamos que, em geral: existe uma diferença positiva entre o TE do último comentário e o do post; o TE do último comentário do autor da thread é maior que o do post que a iniciou e superior à média do TE dos comentários da árvore de discussão.

2. Modelos para Previsão do Tom Emocional dos Usuários. A partir das análises do TE dos usuários, propomos modelos para prever a evolução do TE dos participantes destas comunidades. Utilizamos o Multilayer Perceptron que consiste em uma rede neural com pelo menos três camadas. Estes modelos capturam com boa acurácia (MSE 0.66) a variação do estado emocional dos usuários (que assume valores entre -2 e 2). Uma possível aplicação dos modelos é auxiliar intervenções promovidas por profissionais da área de saúde em redes sociais.

\footnotetext{
${ }^{3}$ https://www.who.int/mental_health/evidence/atlas/interactive_ infographic_2015.pdf

${ }^{4}$ https://www.reddit.com/

${ }^{5}$ https://redditblog.com/2019/12/04/reddits-2019-year-in-review/
} 
Este artigo está organizado da seguinte forma: a Seção 2 descreve os principais trabalhos relacionados; a Seção 3 detalha os dados e métodos utilizados; os resultados da análise do tom emocional e dos modelos são apresentados na Seção 4; as implicações deste trabalho e trabalhos futuros são discutidos na Seção 5.

\section{Trabalhos Relacionados}

Com o passar dos anos, asn Redes Sociais Online (RSO) passaram a permitir o compartilhamento de vários tipos de mídia, resultando em um grande número de trabalhos na literatura que utilizam dados oriundos destas redes para auxiliar o comportamento dos usuários em diferentes aspectos da vida cotidiana. Por exemplo, podemos citar o estudo realizado por [Cunha et al. 2017] que propõe um framework para relevar relações de causalidade entre interações de usuários no subreddit "loseit" e perda de peso.

Além da saúde física, alguns trabalhos na literatura utilizam RSOs como ferramenta para entender problemas relacionados à saúde mental. Os autores em [Blair and Abdullah 2018] utilizam dados do Instagram para identificar e analisar os desafios enfrentados pelos usuários que divulgam seus problemas de saúde mental nesta rede. O trabalho mostra que a mídia social pode ter um impacto positivo para esses usuários, uma vez que permite que eles construam suas próprias comunidades. O trabalho feito por [Islam et al. ] utiliza o Facebook como objeto de estudo para detecção de depressão através de técnicas de aprendizado de máquina. Em [Gruda and Hasan 2019, Sahota and Sankar 2019, Silveira et al. 2018], os autores abordam a detecção da ansiedade, bipolaridade, depressão e suicídio em diferentes redes sociais e procuram entender o comportamento dos usuários presentes nessas redes sociais como forma de propor políticas que contribuam para a diminuição do volume de pessoas afetadas por estes problemas. Considerando o Reddit, os autores em [De Choudhury and De 2014] utilizam dados coletados desta rede para analisar os posts e comentários dos usuários, investigando como o grau de desinibição nos comentários e posts feitos por usuários anônimos se difere daqueles feitos pelos usuários que se identificam.

A maior parte dos trabalhos listados anteriormente tem como foco analisar o comportamento das pessoas que sofrem de doenças relacionadas à saúde mental, visando auxiliar a elaboração de políticas de saúde pública, desenvolver aplicações que auxiliem estas pessoas, entre outras medidas. Diferentemente dos trabalhos listados, neste artigo analisamos o tom emocional de usuários de quatro comunidades online relacionadas a transtornos mentais: depressão, suicídio, ansiedade e bipolaridade, com o principal objetivo de mensurar o impacto que as interações nessas comunidades geram no estado de seus usuários. Nosso estudo analisa o usuário desde o momento em que publica um post até o momento em que realiza seu último comentário dentro de cada thread, permitindo a análise da evolução de seu tom emocional e de suas interações dentro da comunidade. A partir da análise que realizamos, propomos modelos que podem ser utilizados para prever tom emocional dos usuários e que podem ser futuramente utilizados para auxiliar intervenções promovidas por profissionais da área de saúde em redes sociais.

\section{Metodologia}

Nesta seção, apresentamos: (i) a metodologia para analisar como as interações através de posts e comentários influenciam o estado emocional de comunidades de saúde mental 
e (ii) os conceitos e modelos para prever a variação do tom emocional dos usuários que participam destas comunidades.

Conjunto de Dados. Existem mais de 25 subreddits que focam na discussão de transtornos mentais. Os quatro com o maior número de posts e comentários [Gkotsis et al. 2016] são ${ }^{6}$ : Depression (/r/depression), SuicideWatch (/r/suicide), Anxiety (/r/Anxiety) e Bipolar (/r/bipolar). Neste trabalho, utilizamos todos os posts e comentários compartilhados nestas comunidades entre Janeiro de 2011 e Dezembro de $2017^{7}$.

A Tabela 1 apresenta estatísticas básicas agregadas sobre o período selecionado. Consideramos todos posts e comentários, inclusive aqueles que foram removidos, deletados ou estavam vazios. Nas estatísticas apresentadas, desconsideramos os posts e comentários realizados por usuários deletados (i.e., usuários que foram excluídos do Reddit), porque não conseguiríamos distinguir os autores destas publicações. Podemos notar que a comunidade Bipolar é a que realiza, proporcionalmente, mais posts e comentários. Além disso, a média dos comentários por usuário nesta comunidade é cerca de três vezes maior que nas outras três. Isto é um indício que os usuários nesta comunidade são mais engajados durante as discussões.

\begin{tabular}{|l|r|r|r|r||r|}
\cline { 2 - 6 } \multicolumn{1}{c|}{} & Depression & SuicideWatch & Anxiety & Bipolar & Total \\
\hline Usuários Únicos & 333.624 & 162.363 & 121.945 & 35.081 & 569.122 \\
\hline Posts & 468.507 & 169.541 & 142.168 & 73.254 & 853.470 \\
\hline Comentários & 2.128 .991 & 1.024 .171 & 685.867 & 607.262 & 4.446 .291 \\
\hline \hline Posts/usuário & 1,40 & 1,04 & 1,17 & 2,09 & 1,51 \\
\hline Comentários/usuário & 6,38 & 6,31 & 5,62 & 17,31 & 7,81 \\
\hline Comentários/post & 4,54 & 6,04 & 4,82 & 8,29 & 5,21 \\
\hline
\end{tabular}

Tabela 1. Estatísticas básicas de cada comunidade.

Análise Textual. Tom emocional é o sentimento (combinação do positivo e negativo) extraído através de um texto. Assim, para mensurar o estado de um usuário, analisamos o texto dos posts e comentários coletados utilizando a ferramenta de análise de sentimentos Vader [Hutto and Gilbert 2014]. Após a execução do algoritmo, o texto analisado é classificado, proporcionalmente, nas seguintes categorias léxicas: positiva, negativa ou neutra. Adicionalmente, a ferramenta calcula a composição dos três valores léxicos normalizados entre -1 (extremo negativo) e 1 (extremo positivo), denominado compound, a que se refere por tom emocional.

Análise do Tom Emocional. A análise do tom emocional e a quantificação da sua variação ao longo do tempo são realizadas considerando as árvores de discussão extraídas das interações entre os usuários dos subreddits considerados. Definimos como árvore de discussão uma thread inciada pelo post de um usuário, seguido por um ou mais comentários. Para analisarmos a variação do tom emocional em cada árvore de discussão isoladamente, consideramos apenas sequências de comentários em que: (i) exista pelo menos uma interação com outro usuário e (ii) o intervalo entre duas interações consecutivas seja menor ou igual a 24 horas.

A Figura 1 ilustra a metodologia para seleção das árvores de discussão. O eixo

\footnotetext{
${ }^{6}$ Não consideramos o subreddit Opiates (/r/opiates) por estar mais ligado à dependência de remédios.

${ }^{7}$ Os dados foram recuperados em: http://files.pushshift.io/reddit/
} 
horizontal corresponde ao tempo. O usuário em questão realiza atividades (posts e/ou comentários) nas threads 1 a 5 durante o intervalo ilustrado. As atividades realizadas por este usuário são marcadas por ticks mais grossos, identificados por $\mathrm{P}$ no caso de post, ou $\mathrm{C}_{i}$ no caso do $i$-ésimo comentário que ele faz na thread. Atividades de outros usuários são marcadas pelos ticks mais finos. No exemplo, a thread 4 não será selecionada para análise porque entre o post e seu primeiro comentário na thread, ele interagiu na thread 2. Por razão análoga, o comentário $c_{2}$ na thread 2 será desconsiderado. Dado que a ordem em que as threads são avaliadas impacta no conjunto final de árvores de discussão, as threads são ordenadas de forma crescente segundo a data de criação do post. Após aplicar o critério de seleção apresentado, permanecemos com 87.906 árvores no Depression (27,78\% do total), 41.087 no SuicideWatch (37,81\% do total), 33.821 no Anxiety (32,64\% do total) e 19.267 no Bipolar (33,17\% do total).

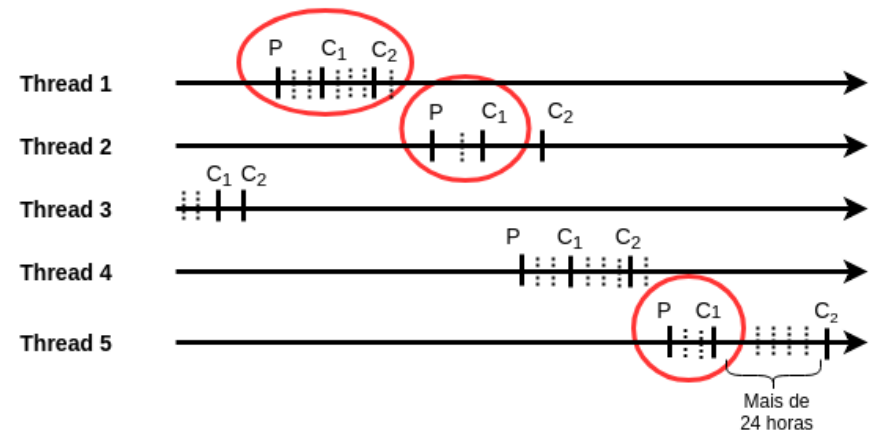

Figura 1. Apenas os trechos das árvores de discussão circulados foram considerados. 0 usuário $A$ inciou as threads $1,2,4$ e 5 . Não consideramos a thread 4, pois o usuário ainda realizou interação na thread 2.

Tarefa de Previsão. A tarefa de previsão do tom emocional de um usuário é definida a seguir. Consideramos como entradas dos modelos de previsão: o post inicial $p$, com tom emocional $\operatorname{TE}(p)$ realizado por um usuário $u$, e o conjunto de comentários da mesma thread $\left(c_{1}, c_{2}, \ldots, c_{\text {last }}\right)$, onde o comentário $c_{\text {last }}$, com tom emocional $\mathrm{TE}\left(c_{\text {last }}\right)$, é o último comentário realizado pelo usuário $u$. O resultado do modelo é uma previsão da variação do tom emocional, $\mathrm{TE}\left(c_{\text {last }}\right)$ - TE $(p)$, do usuário $u$. A partir deste resultado, será possível verificar se houve alguma mudança do sentimento do usuário após as interações realizadas com os demais usuários da comunidade em questão.

Representação dos Posts e Comentários. Para desenvolver a tarefa proposta, é preciso representar posts e comentários através de atributos. Neste trabalho, utilizamos a técnica de Word Embeddings (WE), também conhecida como words vectors ou word representations, que representam palavras como vetores em um espaço latente. Nesse espaço, vetores similares representam palavras com o significado ou função semelhantes.

Para a criação dos words-embeddings usamos todos os posts e comentários de 2010 a 2017 de cada subreddit. No pré-processamento, utilizamos a ferramenta ekphrasis $^{8}$, a qual realiza normalização das palavras e correção ortográfica. Aplicamos a normalização no e-mail, telefones e horas. Também removemos as pontuações, caracteres especiais e excesso de espaço. Para remover as stopwords utilizamos o Natural Language Toolkit (NLTK).

\footnotetext{
${ }^{8}$ https://github.com/cbaziotis/ekphrasis
} 
Na geração dos Words Embeddings (WE) utilizamos o pacote Gensim do Python. Os parâmetros para execução do modelo foram: arquitetura SkipGram; contexto igual à 5; considerando todas as palavras (que tenham aparecido ao menos uma vez no corpus); e dimensão dos vetores 300. O WE retorna um vetor para cada palavra do post/comentário, treinado com o corpus que possuímos. Para agregar os embeddings associados a cada publicação, tomamos a média dos embeddings das palavras. Portanto, cada publicação é representada por um vetor composto pela média dos embeddings de todas as palavras.

Modelos de Previsão. Neste trabalho, utilizamos o modelo de rede neural Multilayer Perceptron (MLP) para prever o tom emocional dos usuários das comunidades analisadas. Escolhemos esse modelo por capturar possíveis relações não-lineares entre o TE e pares de features (o que não seria possível com uma regressão linear), sem o elevado número de parâmetros de uma arquitetura mais profunda. Utilizamos 3 camadas ocultas, uma com 128 neurônios, as outras duas com 256. Foram criadas dois tipos de redes neurais: (i) uma apenas com perceptrons e (ii) outra com camadas de regularização. Para a regularização utilizamos a camada de Dropout com os valores de 0.3, 0.5 e 0.7. Depois dos testes, permanecemos com o valor de 0.5 . Consideramos 5 variações do conjunto de atributos de entrada para cada uma das redes apresentadas, discutidas a seguir. É importante ressaltar que em todos modelos, desconsideramos o último comentário do autor do post na árvore de discussão ao calcular o vetor de entradas para a rede (i.e., as features).

- Modelo 1 - Agregação de comentários (dimensão da entrada = 300): a entrada é um vetor de dimensão 300 que é o embedding médio de todos os comentários da árvore de discussão.

- Modelo 2 - Agregação de Post e Comentários (dimensão da entrada = 300): a entrada é um vetor de dimensão 300 que é a média do embedding do post com o embedding médio de todos comentários da árvore de discussão.

- Modelo 3 - Post e agregação de Comentários (dimensão da entrada =600): a entrada é composta por dois vetores concatenados, sendo um vetor de dimensão 300 que o embedding médio de todos comentários da árvore de discussão, e outro vetor de dimensão 300 que é o embedding do post.

- Modelo 4 - Post, agregação de comentários do autor e agregação de outros comentários (dimensão da entrada $=900$ ): a entrada é composta por três vetores, sendo um vetor de dimensão 300 que é o embedding médio de todos comentários (excluindo aqueles do usuário que realizou o post), outro vetor de dimensão 300 que é o embedding médio dos comentários do autor do post, e um vetor de dimensão 300 que é o embedding do post.

- Modelo 5 - Agregação de post e comentários do autor e agregação de outros comentários (dimensão da entrada $=600$ ): a entrada é composta por dois vetores, sendo um vetor de dimensão 300 que é o embedding médio de todos comentários (excluindo aqueles do usuário que realizou o post); outro vetor de dimensão 300 igual a média entre o embedding do post e o embedding médio dos comentários do autor do post.

A avaliação da acurácia dos diferentes modelos é realizada a partir da métrica de MSE (Mean Squared Error). 


\section{Resultados}

Nesta Seção apresentamos os resultados da análise do tom emocional dos usuários e dos modelos de predição propostos.

\subsection{Análise do Tom Emocional}

Primeiramente, introduzimos a notação que será utilizada nesta análise. Seja $\operatorname{TE}(p)$ o tom emocional de um post $\mathrm{p}$; $\mathrm{TE}\left(c_{\text {last }}\right)$ o tom emocional do comentário feito pelo autor de uma thread naquela mesma thread; e TE $\left(c_{\text {all }}\right)$ comentários dos outros usuários, excluindo o autor do post.

Características TE Árvores de Discussão. A Figura 2 apresenta a distribuição de, $\mathrm{TE}(p), \operatorname{TE}\left(c_{\text {last }}\right)$ e $\overline{\mathrm{TE}\left(c_{\text {all }}\right)}$, i.e, TE do post, do último comentário do autor do post e a média de TE dos comentários da árvore de discussão, sem incluir $c_{l a s t}$. O gráfico apresentado é do tipo violino, semelhante ao boxplot, mas cuja diferença é a possibilidade de se analisar a densidade dos valores: quanto mais largo o formato, maior é a concentração de pontos naquela região. O ponto branco indica a mediana. A barra mais grossa é o intervalo interquartil.

É possível observar que, em todos subreddits, o $1^{\circ}$ quartil e a mediana referentes ao post estão bem abaixo das respectivas medianas dos comentários dos últimos comentários do autor e dos comentários dos outros usuários. Além disso, o $3^{\circ}$ quartil referente ao post também se encontra abaixo daquele referente à média dos comentários, exceto no subreddit Bipolar. Em geral, os comentários tendem a ser mais positivos que os posts, exceto para o subreddit Bipolar, que apresenta também um grande volume de posts com o tom emocional mais positivo. Note que, em geral, há maior concentração de posts na região de TE negativo. Por outro lado, no caso do último comentário feito pelo autor da thread, há maior concentração na região de TE positivo.

Além disso, a mediana do TE médio dos comentários (i.e., de $\left.\overline{\mathrm{TE}\left(c_{\text {all }}\right)}\right)$ é menor que o TE do último comentário do autor do post, exceto no subreddit SuicideWatch. Esta análise sugere que as interações do autor de um post com outros usuários, ao receber comentários com TE positivo, podem contribuir para o aumento do seu TE.

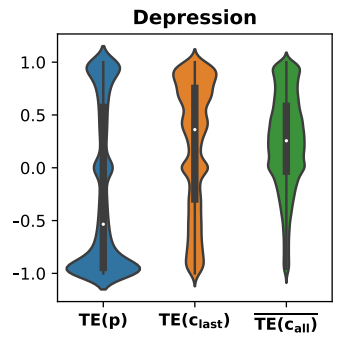

(a) Depression

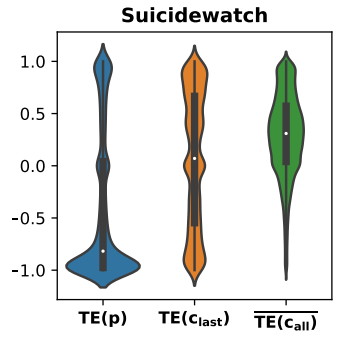

(b) Suicide

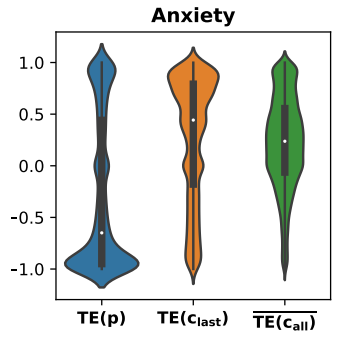

(c) Anxiety

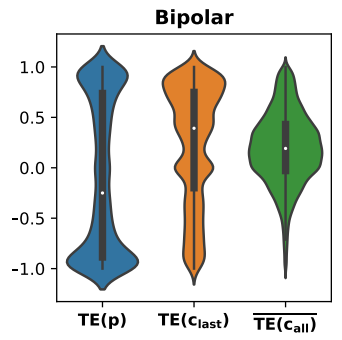

(d) Bipolar

Figura 2. Tom Emocional.

Relação TE do Post com os Comentários. Para entender como os usuários que participam de uma árvore de discussão interagem com o autor do post, apresentamos a Figura 3, que mostra um mapa de calor do número de árvores de discussão, agrupadas de acordo com o TE do post (eixo x) e com o TE médio dos comentários da árvore de discussão, 
excluindo-se o post inicial. É possível observar que quando o post tem TE extremamente negativo (-1), a média dos comentários dos outros usuários na árvore de discussão tende a ser mais positiva (valores acima 0 ).

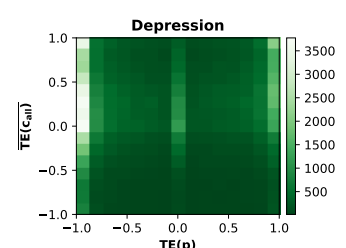

(a) Depression

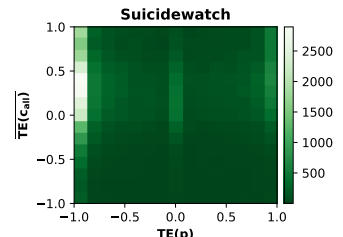

(b) Suicide

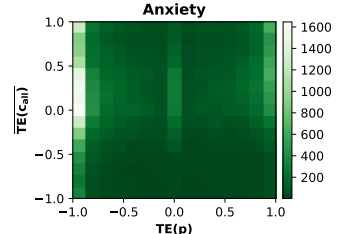

(c) Anxiety

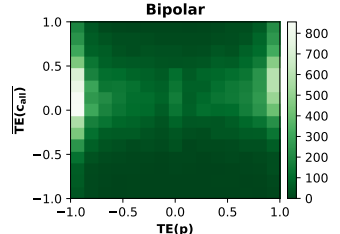

(d) Bipolar

Figura 3. Tom Emocional dos Posts x Tom Emocional Médio dos Comentários.

Relação TE do Post com o Último Comentário. Averiguamos também se ocorre mudança do TE entre o momento em que um usuário escreve um post e o momento em que faz seu último comentário na árvore de discussão, utilizando um mapa de calor semelhante ao anterior, mostrado na Figura 4. Note que, autores de posts extremamente negativos (-1) tendem a escrever comentários mais positivos ao final da thread. Esta variação sugere que os comentários realizados por outros usuários podem ajudar os usuários que se encontram em situações difíceis.

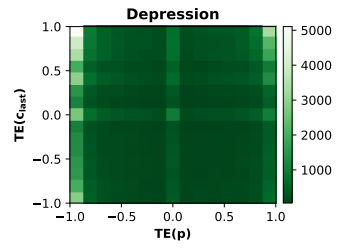

(a) Depression

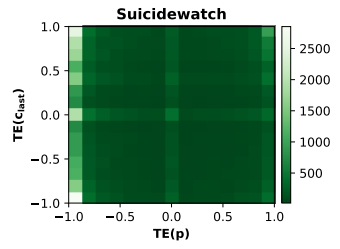

(b) Suicide

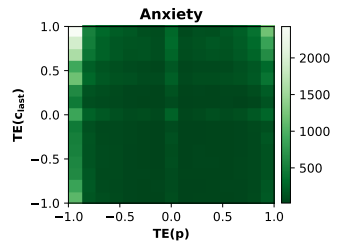

(c) Anxiety

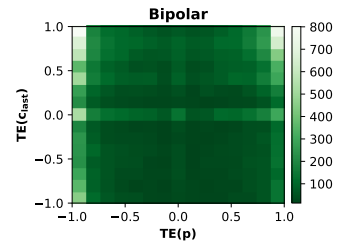

(d) Bipolar

Figura 4. Tom Emocional dos Posts $x$ Tom Emocional do Último Comentário.

Relação TE do Último Comentário com os demais Comentários. O passo seguinte foi investigar se o TE do último comentário do autor tem relação com a média do TE dos comentários da árvore de discussão (excluindo-se o post inicial), calculando-se o mapa de calor mostrado na Figura 5. Note que, em todos subreddits, um TE positivo no último comentário do autor do post está relacionado a um TE positivo médio entre os comentários thread. No SuicideWatch também ocorre uma grande quantidade de comentários negativos por parte dos autores da thread, mesmo quando estão relacionados a uma árvore de discussão com TE positivo. Isto pode ser um indício de que existem usuários nesta comunidade que a melhoria do tom emocional, ou seja, o aumento da positividade nos textos das suas atividades, é mais difícil de ocorrer.

Relação TE dos Comentários com Diferença do TE do último comentário e o Post. Como observado anteriormente, o TE dos comentários das árvores de discussões estão positivamente correlacionados com o TE do último comentário. Consequentemente, podem estar relacionados com uma variação positiva, considerando-se a diferença entre o TE do post e o TE do último comentário. Para investigar melhor estas relações, analisamos a diferença, para cada árvore de discussão, entre o tom emocional do último comentário do autor e do post. A Figura 6 apresenta a distribuição desta diferença. Observe que ocorre 


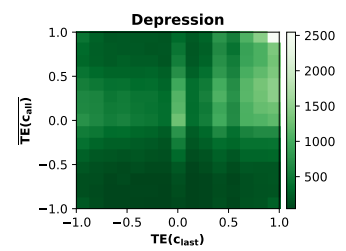

(a) Depression

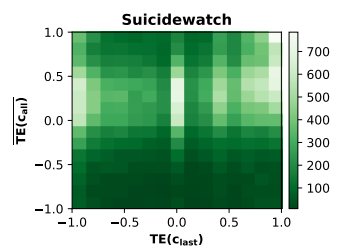

(b) Suicide

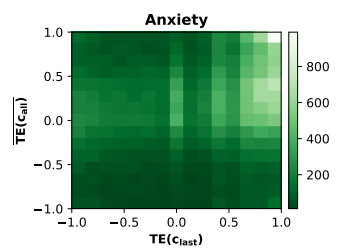

(c) Anxiety

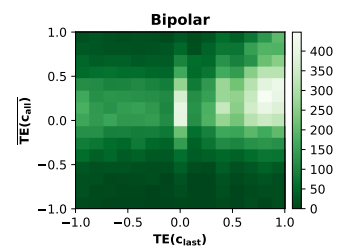

(d) Bipolar

Figura 5. Média do Tom Emocional dos Comentários x Tom Emocional do último comentário

maior concentração nos valores acima de zero, mostrando que o usuário, na maioria das vezes, "melhora" ao final da thread, em relação ao TE. Analisamos então a relação entre a média do TE dos comentários e a variação do TE observada pelo autor do post. Na Figura 7, notamos que quando a média é positiva, existe uma diferença positiva entre o TE do último comentário e o post, a qual pode ser observada no canto superior direito. Note que quando a média é negativa, não existe um padrão claro para a diferença do TE.

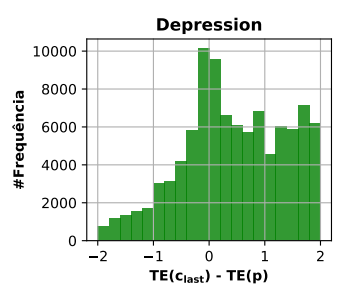

(a) Depression

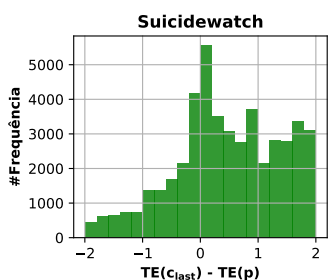

(b) Suicide

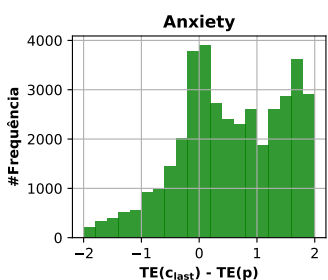

(c) Anxiety

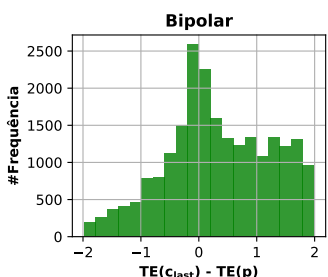

(d) Bipolar

Figura 6. Histograma da Diferença do Tom Emocional entre comentário e post

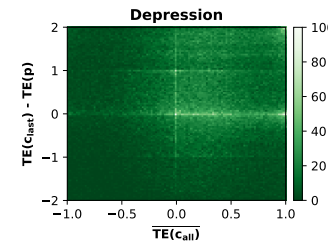

(a) Depression

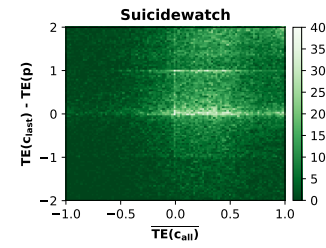

(b) Suicide

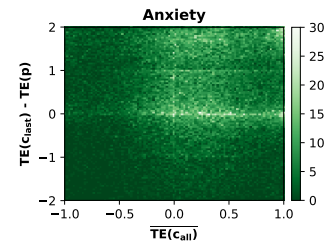

(c) Anxiety

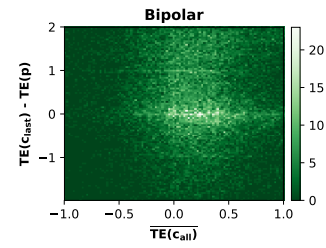

(d) Bipolar

Figura 7. Média do Tom Emocional dos Comentários x Diferença do tom emocional entre último comentário e post.

Resumindo, as análises realizadas fornecem um conjunto de observações relevantes a respeito da dinâmica do tom emocional de um usuário ao participar em uma árvore de discussão. Investigamos vários aspectos do TE dentro da árvore de discussão: nos posts, nos comentários, no último comentário do autor do post e na diferença do TE. Um dos resultados mais importantes é a existência da correlação entre o último comentário do usuário que realizou a postagem com os comentários dos demais usuários na árvore de discussão. Além disso, analisamos que um comentário influencia o TE do próximo comentário, um indício de que o aspecto temporal pode impactar na evolução do comportamento do usuário dentro da comunidade. A seguir, avaliamos a acurácia do modelo para prever a diferença do TE do usuário ao longo tempo, dentro da árvore de discussão. 


\subsection{Previsão do Tom Emocional}

Nesta seção apresentamos os resultados da acurácia dos modelos de previsão propostos.

Baselines. Definidos três baselines para avaliar o quão efetivos são os modelos propostos. Seja $D$ a variável aleatória que representa a variação do TE sofrida pelo autor de uma árvore de discussão escolhida uniformemente ao acaso, definida por $D=$ $\mathrm{TE}\left(c_{\text {last }}\right)-\mathrm{TE}(p)$. Sejam ainda $D_{i}$ a variação do TE associada à thread $i$ e $\hat{D}_{i}$ a respectiva previsão, retornada por um dado modelo. Considerando o Mean Squared Error (MSE) como medida de erro, podemos avaliar os modelos utilizando a equação

$$
\mathrm{MSE}=\frac{1}{n} \sum_{i=1}^{n}\left(D_{i}-\hat{D}_{i}\right)^{2}
$$

onde as variáveis $D_{1}, \ldots, D_{n}$ representam a variação de TE associada às threads do conjunto de teste. Para calcular as métricas de erro, utilizamos cross-validation com 5 folds.

Os resultados dos modelos propostos neste artigo são comparados com os resultados obtidos pelos seguintes baselines:

- Baseline 1: O primeiro baseline é o modelo que sempre retorna 0 para a variação do TE, ou seja, que assume que o usuário manteve seu TE na árvore de discussão. Neste caso, o MSE do Baseline 1 é dado por: $\operatorname{MSE}\left(B_{1}\right)=E\left[D^{2}\right]$.

- Baseline 2: O segundo baseline é o modelo que sempre retorna a variação média do TE, $\bar{D}=\frac{1}{n} \sum_{i=1}^{n} D_{i}$. Neste caso, o erro de previsão é calculado por: $\operatorname{MSE}\left(B_{2}\right)=E\left[(D-\bar{D})^{2}\right]$, ou seja, é a variância de $D$.

- Baseline 3: O terceiro baseline utilizado foi uma regressão linear considerando como atributos o total de comentários e a média do TE da árvore de discussão (excluindo-se àqueles de quem fez o post) para prever a diferença do TE. O erro correspondente é calculado através da Eq. 1.

A Tabela 2 apresenta o MSE obtido para cada baseline avaliado em cada comunidade. Observe que os MSE's do baseline 3 são menores que os MSE's dos baselines 1 e 2, mas que a diferença em relação ao modelo 2 é pequena, indicando a dificuldade de prever $D$ a partir de $\overline{\mathrm{TE}\left(c_{\text {all }}\right)}$. Assim, nossos modelos terão o objetivo apresentar resultados mais precisos que os resultados do baseline 3 .

\begin{tabular}{|l|r|r|r|}
\cline { 2 - 4 } \multicolumn{1}{c|}{} & \multicolumn{3}{c|}{ Mean Squared Error (MSE) } \\
\hline Subreddit & Baseline 1 & Baseline 2 & \multicolumn{1}{c|}{ Baseline 3 } \\
\hline Depression & 1,023 & 0,854 & 0,848 \\
\hline SuicideWatch & 1,029 & 0,843 & 0,839 \\
\hline Anxiety & 1,062 & 0,818 & 0,813 \\
\hline Bipolar & 0,964 & 0,819 & 0,811 \\
\hline
\end{tabular}

Tabela 2. MSE's dos Baselines para cada comunidade.

Avaliação. Testamos cada modelo com e sem regularização, a Tabela 3 apresenta os resultados das 10 configurações ( 5 modelos $\times$ uso/não-uso de regularização) para a comunidade Bipolar. Observe que os resultados dos modelos com e sem regularização são semelhantes. Entretanto, utilizar a regularização é importante para evitar overfitting. Assim escolhemos, dentre os modelos com regularização, o modelo 4, por resultar no menor MSE dentre os demais, sendo inclusive bem menor que o do baseline 3. Em suma, executamos todos os modelos para a comunidade Bipolar e quando encontramos o melhor modelo, aplicamos para as demais comunidades. Note que este modelo é o que mais 
distingue dentre os tipos de publicação, uma vez que existe uma dimensão para os comentários dos usuários que não realizaram o post, outra para os comentários de quem fez o post e outra para o post. Isto mostra que separar os textos em diferentes vetores de entrada é importante para a previsão da diferença do TE.

A única diferença do modelo 5 para modelo 4 é que, no modelo 5 , o vetor do embedding médio dos comentários do autor do post $\mathrm{e}$ o vetor dos embedding do seu post são agregados em um único vetor, que possui características textuais do mesmo autor. Esta diferença ocasiona um erro 8.8\% maior do modelo 5. Apesar desses textos terem sido realizados pelo mesmo autor, é importante distinguir entre post e comentários do autor para melhor prever a variação do TE. Isto ocorre possivelmente devido à mudança no discurso dos comentários do autor do post, influenciado pelos demais usuários.

Aplicamos o modelo 4 às demais comunidades, Depression, SuicideWatch e Anxiety. A Tabela 4 apresenta os resultados. A maior diferença percentual no MSE entre comunidades é de apenas 1,02\% (entre as comunidades Bipolar e Depression), mostrando que os resultados para as quatro comunidades são similares. Em comparação com o Baseline 3, o modelo 4 obteve melhores resultados de acurácia para todas as comunidades: na comunidade Depression, a diminuição do MSE é de 20,79\%, no SuicideWatch 20,04\%, no Anxiety 18,02\% e no Bipolar 18,02\%. Uma possível explicação para o melhor desempenho do modelo 4 na tarefa de previsão proposta, está na separação entre o post, os comentários do autor da thread e os comentários dos outros usuários, isolando diferentes tipos de tons emocionais inerentes às atividades consideradas: por exemplo, o post tem o tom mais negativo e normalmente é um pedido de ajuda, os comentários dos outros usuários costumam ter o tom mais positivo e tem o papel de oferecer suporte e conselhos, já os comentários do autor do post podem refletir um processo de melhora.

\begin{tabular}{|c|r|r|r|r|}
\cline { 2 - 5 } \multicolumn{1}{c|}{} & \multicolumn{2}{c|}{ Sem Regularizaç̃̃o } & \multicolumn{2}{c|}{ Com regularização } \\
\cline { 2 - 5 } \multicolumn{1}{c|}{} & Media MSE & \multicolumn{1}{c|}{ DP MSE } & Media MSE & \multicolumn{1}{c|}{ DP MSE } \\
\hline Modelo 1 & 0,8186 & 0,0236 & 0,8061 & 0,0192 \\
\hline Modelo 2 & 0,784 & 0,0182 & 0,7844 & 0,0186 \\
\hline Modelo 3 & 0,671 & 0,0084 & 0,6961 & 0,0446 \\
\hline Modelo 4 & 0,6781 & 0,0205 & 0,6651 & 0,0191 \\
\hline Modelo 5 & 0,7174 & 0,0121 & 0,7295 & 0,0203 \\
\hline
\end{tabular}

Tabela 3. Resultados MSE das variações dos Modelos MLP's para a comunidade Bipolar. Baseado em 5 folds em termos de média e desvio padrão.

\begin{tabular}{|l|r|r|}
\hline Subreddit & Media MSE & DP MSE \\
\hline Depression & 0,6719 & 0,0225 \\
\hline SuicideWatch & 0,6707 & 0,02568 \\
\hline Anxiety & 0,6665 & 0,0264 \\
\hline Bipolar & 0,6651 & 0,0191 \\
\hline
\end{tabular}

Tabela 4. Resultados do MSE Modelo MLP. Baseado em 5 folds em termos de média e desvio padrão.

Em suma, os modelos propostos neste trabalho são eficazes para capturar a variação do TE do usuário em uma árvore de discussão. Esses modelos podem ser utilizados no monitoramento de conversas, por exemplo, em redes sociais de suporte a usuários com problemas de saúde mental. Ao identificar que o usuário não está melhorando seu tom emocional, especialistas podem intervir para evitar situações extremas.

\section{Conclusão}

As análises apresentadas neste trabalho mostram que as interações entre os usuários de RSOs relacionadas aos transtornos de saúde mental auxiliam na melhoria das suas condições de saúde, uma vez que o TE dos usuários tendem a sofrer variações positivas 
ao longo das interações realizadas. Com base nesta análise, propomos modelos que preveem com boa acurácia a variação do TE dos usuários participantes destas comunidades.

Como os diversos trabalhos que utilizam dados de redes sociais online, nosso trabalho possui como principal limitação a impossibilidade de verificar se uma variação positiva no TE está correlacionada com uma melhora real no estado emocional do usuário, já que não temos um contato direto com usuário para averiguação. No entanto, nossos modelos podem ser aplicados para monitorar a evolução do tom emocional de tais usuários durante as discussões realizadas nestas comunidades: caso o TE não esteja evoluindo positivamente, intervenções podem ser realizadas por profissionais da área, evitando situações extremas (por exemplo, o suicídio).

\section{Referências}

[Barney et al. 2006] Barney, L. J., Griffiths, K. M., Jorm, A. F., and Christensen, H. (2006). Stigma about depression and its impact on help-seeking intentions. Australian \& New Zealand Journal of Psychiatry, 40(1):51-54.

[Blair and Abdullah 2018] Blair, J. and Abdullah, S. (2018). Supporting constructive mental health discourse in social media. In PervasiveHealth ' 18.

[Cunha et al. 2017] Cunha, T., Weber, I., and Pappa, G. (2017). A warm welcome matters!: The link between social feedback and weight loss in/r/loseit. In WWW'17 Companion, pages 1063-1072. International World Wide Web Conferences Steering Committee.

[De Choudhury 2013] De Choudhury, M. (2013). Role of social media in tackling challenges in mental health. In Proceedings of the 2nd international workshop on Sociallyaware multimedia, pages 49-52. ACM.

[De Choudhury and De 2014] De Choudhury, M. and De, S. (2014). Mental health discourse on reddit: Self-disclosure, social support, and anonymity. In ICWSM.

[Gkotsis et al. 2016] Gkotsis, G., Oellrich, A., Hubbard, T., Dobson, R., Liakata, M., Velupillai, S., and Dutta, R. (2016). The language of mental health problems in social media. In Proceedings of the Third Workshop on Computational Lingusitics and Clinical Psychology.

[Gruda and Hasan 2019] Gruda, D. and Hasan, S. (2019). Feeling anxious? perceiving anxiety in tweets using machine learning. Computers in Human Behavior, 98:245255 .

[Hutto and Gilbert 2014] Hutto, C. J. and Gilbert, E. (2014). Vader: A parsimonious rulebased model for sentiment analysis of social media text. In ICWSM.

[Islam et al. ] Islam, M. R., Kabir, M. A., Ahmed, A., Kamal, A. R. M., Wang, H., and Ulhaq, A. Depression detection from social network data using machine learning techniques. Health Information Science and Systems.

[Sahota and Sankar 2019] Sahota, P. K. and Sankar, P. L. (2019). Bipolar disorder, genetic risk, and reproductive decision-making: A qualitative study of social media discussion boards. Qualitative health research.

[Silveira et al. 2018] Silveira, B., da Silva, A. P. C., and Murai, F. (2018). Análise de comunidades de suporte a transtornos de saúde mental do reddit. In Anais do VII Brazilian Workshop on Social Network Analysis and Mining. SBC. 